\title{
The ground state of a mixture of two species of fermionic atoms in 1D optical lattice
}

\author{
Shi-Jian Gu, Rui Fan, and Hai-Qing Lin \\ Department of Physics and Institute of Theoretical Physics, \\ The Chinese University of Hong Kong, Hong Kong, China
}

(Dated: September 17, 2018)

\begin{abstract}
In this paper, we investigate the ground state properties of a mixture of two species of fermionic atoms in one-dimensional optical lattice, as described by the asymmetric Hubbard model. The quantum phase transition from density wave to phase separation is investigated by studying both the corresponding charge order parameter and quantum entanglement. A rigorous proof that even for the single hole doping case, the density wave is unstable to the phase separation in the infinite $U$ limit, is given. Therefore, our results are quite instructive for both on-going experiments on strongly correlated cold-atomic systems and traditional heavy fermion systems.

PACS numbers: 03.75.Gg, 71.10.Fd, 03.75.Mn, 05.70.Jk
\end{abstract}

\section{INTRODUCTION}

Rapid progress in Bose-Einstein condensates in optical lattices 1, 2, 3, 4, 5] has opened fascinating experimental possibilities in condensed matter physics, atomic physics, and quantum information. For example, the experiment on neutral atoms trapped in the periodic potential of an optical lattice has been used to realize an array of quantum gates [4]. Moreover, cold-atomic systems are intrinsically related to many-body models in condensed matter physics. Compared to solid state systems, coldatomic systems could be better experimentally controllable. Thus, the investigation of cold-atomic systems will not only help us to have a deep understanding of known physical phenomena in many-body systems, but also provide hints to explore new areas of physics. Such a beautiful prospect has attracted many theoretical attentions $6,7,8,9,10,11$.

Quite recently, experiments on fermionic atoms trapped in optical lattices [5] were carried out which opened a door for us to find deeper insights into some essential problems in condensed matter physics, such as BEC-BCS crossover, superfluidity, and Mott-insulating phase. It was proposed that ultracold fermionic atoms exposed to the periodical potential of an optical lattice could be an ideal realization of the Bose-Hubbard model [6], the spin-dependent Hubbard model [7], and the antiferromagnetic states or $d$-wave pairing states [8]. The unique control over all relevant parameters in these systems [5] allows people to carry out experiments which are not handy with solid state systems, so they marked a milestone towards the understanding of some fundamental concepts in quantum many-body systems.

In this paper, we consider a system of two species of fermionic atoms 9] with equal numbers (or one type of fermionic atoms with spin-depent hoping integral[7]) away from half-filling in an one-dimensional optical lattice, as described by the asymmetric Hubbard model. [12, 13, 14] (AHM). The system is expected to have a density wave (DW) state and phase separation (PS) of two atom species state [15], and we investigate the quantum phase transition (QPT) from the DW state to the
PS state in this system by studying both the quantum entanglement and traditional DW order parameter. We show that the entanglement can help us to witness critical phenomenon and shows scaling behavior around the critical point. The phase transition is also clarified by the competition between two different modes of structure factor. A global phase diagram as a function of the local interaction $U$ and the ratio of two hoping integrals is then obtained under different filling conditions. Moreover, we give a rigorous proof, that even for the case of a single hole doping away from half-filling, the DW state is unstable to the PS state in the infinite $U$ limit. As will be shown below, if we regard two regions in the PS phase as one solid-like region of heavy atoms and another as a liquid-like region of light atoms, respectively, the QPT is just a physical realization of the quantum solvation process [16] in the optical lattice. Therefore, our results are quite instructive for on-going experiments on strongly correlated cold-atomic systems. The behavior of entanglement in this system can help people to have a deep understanding of the critical phenomenon.

This paper is organized as follows. In section II we introduce the Hamiltonian of the AHM, and show how to realize the model in the quasi one-dimensional periodical potential of an optical lattice. We will also briefly introduce the background of the model in the condensed matter physics. In section [II] we study the groundstate entanglement of the system. We will show that a schematic phase diagram can be obtained from the entanglement between a local part and the rest of the system of a finite sample. In section IV by studying the structure factor of the density distribution of heavy atoms, we can obtain a quantitative phase diagram for different filling conditions via both the exact diagonalization (ED) and density matrix renormalization group (DMRG) methods. In section $\mathrm{V}$, we will give a rigorous proof that even for the case of a single hole doping away from half-filling, the DW state is unstable to the PS state in the infinite $U$ limit. While if $U$ is very large, the critical point then is approached linearly with $1 / U$. In section VI, we will discuss the mechanism of the existence of the PS, the possibility of the PS in high dimension, and conditions 
for experimental realization. Finally, we summarize our results in section VII

\section{THE MODEL HAMILTONIAN}

The one-dimensional AHM is defined as

$$
H=-\sum_{j=1}^{L} \sum_{\delta= \pm 1} \sum_{\sigma} t_{\sigma} c_{j, \sigma}^{\dagger} c_{j+\delta, \sigma}+U \sum_{j=1}^{L} n_{j, \alpha} n_{j, \beta} .
$$

In Eq.(1), $t_{\sigma}(\sigma=\alpha, \beta)$ distinguishes the species of fermionic atoms (e.g., ${ }^{6} \mathrm{Li}$ and ${ }^{40} \mathrm{~K}$ ), $c_{j, \sigma}^{\dagger}$ and $c_{j, \sigma}, \sigma=\uparrow, \downarrow$ are creation and annihilation operators for $\sigma$ atoms at site $j$ respectively, and $n_{\sigma}=c_{\sigma}^{\dagger} c_{\sigma}$, while $U$ denotes the strength of on-site interaction. In this model, the Hamiltonian has $\mathrm{U}(1) \otimes \mathrm{U}(1)$ symmetry for the general $t_{\sigma}$, and the atoms number $N_{\alpha}=\sum_{j} n_{j, \alpha}, N_{\beta}=\sum_{j} n_{j, \beta}$ are conserved respectively. The total number of atoms is given by $N=N_{\alpha}+N_{\beta}$, and the filling factor is $n=N / L$.

The asymmetric Hubbard model (1) can be used as an effective model to describe a mixture of two species of fermionic atoms in an one-dimensional optical lattice. In order to have a quasi one-dimensional system, we suggest that the optical lattice potential takes the form of

$$
\begin{aligned}
& V(x, y, z)=V_{0} \sin ^{2}(k x)+V_{\perp}\left[\sin ^{2}(k y)+\sin ^{2}(k z)\right] \\
& V_{0}=\nu \frac{\hbar^{2} k^{2}}{2 m} \\
& V_{\perp}=\nu_{\perp} \frac{\hbar^{2} k^{2}}{2 m} .
\end{aligned}
$$

Here $k=2 \pi / \lambda$ and $\lambda$ is the wavelength of the laser light, and $V_{0}$ and $V_{\perp}$ denote the maximum potential depth along the $x$ direction and in the $y z$ plane respectively. The potential depth is measured in units of the recoil energy $\hbar^{2} k^{2} / 2 m$. In order to freeze the hoping process in the $y z$ plane we should have $V_{\perp} \gg V_{0}$. For a single atom in the periodic lattice, its wave function is the Bloch state, which is actually a superposition of well localized Wannier state. Therefore, if we restrict ourself to a very low temperature, where the thermal fluctuation cannot excite the atom to the second band, the Wannier state can be approximated by the ground state of a single atom in the potential well. For the present case, the ground state can be written as

$$
\Psi_{0}(x, y, z) \simeq\left(\frac{m \omega_{\perp}}{\pi \hbar}\right)^{1 / 2} e^{-\frac{m \omega_{\perp}}{2 \hbar}\left(y^{2}+z^{2}\right)} \Psi_{0}(x)
$$

where

$$
\begin{aligned}
& \Psi_{0}(x)=\left(\frac{m \omega}{\pi \hbar}\right)^{1 / 4} e^{-\frac{m \omega}{2 \hbar} x^{2}} \\
& \omega_{\perp}=\frac{\hbar k^{2} \sqrt{\nu_{\perp}}}{m}, \quad \omega=\frac{\hbar k^{2} \sqrt{\nu}}{m} .
\end{aligned}
$$

Then the hoping matrix element between the two adjacent sites $i, j$ can be calculated as

$$
t=-\int d \mathbf{r} w_{\sigma}\left(\mathbf{r}-\mathbf{r}_{i}\right)\left(-\frac{\hbar^{2} \nabla^{2}}{2 m}+V\right) w_{\sigma}\left(\mathbf{r}-\mathbf{r}_{j}\right)
$$

which results in the hoping integral along the $x$ direction

$$
t \simeq \frac{\hbar^{2} k^{2}}{2 m}\left(\sqrt{v}+2 \sqrt{v_{\perp}}\right) e^{-\pi^{2} \sqrt{v}} .
$$

Moreover, if two atoms, $\alpha$ and $\beta$ occupy the same site, they will repel each other. The on-site interaction can be approximated with

$$
U \simeq \frac{4 \pi \hbar^{2} a}{\sqrt{m_{\alpha} m_{\beta}}} \int\left|w_{\alpha}(\mathbf{r})\right|^{2}\left|w_{\beta}(\mathbf{r})\right|^{2} d \mathbf{r},
$$

where $a$ is the scattering length. Using the wave function of Eq. (3), we obtain

$$
U \simeq \frac{4 \pi \hbar^{2} a}{\sqrt{m_{\alpha} m_{\beta}}} \frac{k v^{1 / 4}}{\sqrt{\pi}} \frac{k^{2} v_{\perp}^{1 / 2}}{\pi} .
$$

Finally, if we have a system of two species of polarized fermionic atoms in the optical lattice, the hoping integral and the on-site interaction will have the form (in units of $\left.t_{\alpha}\right)$

$$
\begin{aligned}
\frac{t_{\beta}}{t_{\alpha}} & =\frac{m_{\alpha}}{m_{\beta}}, \\
\frac{U}{t_{\alpha}} & =\frac{16 a \sqrt{\pi m_{\alpha} / m_{\beta}}}{\lambda} \frac{v^{1 / 4} v_{\perp}^{1 / 2}}{\left(\sqrt{v}+2 \sqrt{v_{\perp}}\right)} e^{\pi^{2} \sqrt{v}} .
\end{aligned}
$$

Taking $\operatorname{Li}(\alpha)$ and $\mathrm{K}(\beta)$, two species of atoms, as an example and $v_{\perp}=16$, we have

$$
\begin{aligned}
t_{\beta} & \simeq 0.15 \\
U & \simeq \frac{35.87 a}{\lambda} \frac{v^{1 / 4}}{(\sqrt{v}+8)} e^{\pi^{2} \sqrt{v}} .
\end{aligned}
$$

In condensed matter physics, the asymmetric Hubbard model is one of the most simplest two band models which is believed to describe many essential physical properties of strongly correlated systems. To understand the interesting phenomena which may happen in the ground state of the Hamiltonian (1), it is very useful to look into the two limiting cases of Eq. (1). If $t_{\alpha}=t_{\beta}$, the AHM becomes the Hubbard model [17]. In 1D, the Hubbard model can be solved exactly by the Bethe-ansatz method. 18] The wave function and the energy spectra then can be calculated exactly. In the large $U$ limit, the Hubbard model can be approximated by the famous $t-J$ model, in which the spin-spin interaction is of the antiferromagnetic type. Therefore, it is widely accepted that the ground state of the Hubbard model at halffilling shows the spin-density wave. On the other hand, if $t_{\beta}=0$, the AHM becomes Falicov-Kimball model. [19, 20, 21, 22] In 1D, it has been pointed out that the system will segregate into an empty lattice (with no $\beta$ atoms and all $\alpha$ atoms) and a full lattice (with all $\beta$ atoms and no $\alpha$ atoms) in the large $U$ limit when away from the half-filling. Therefore, the two limiting cases of the AHM belong to different universality classes, a phase transition from PS to DW is expected to appear somewhere on the $U-t_{\beta}$ plane. 
(a)

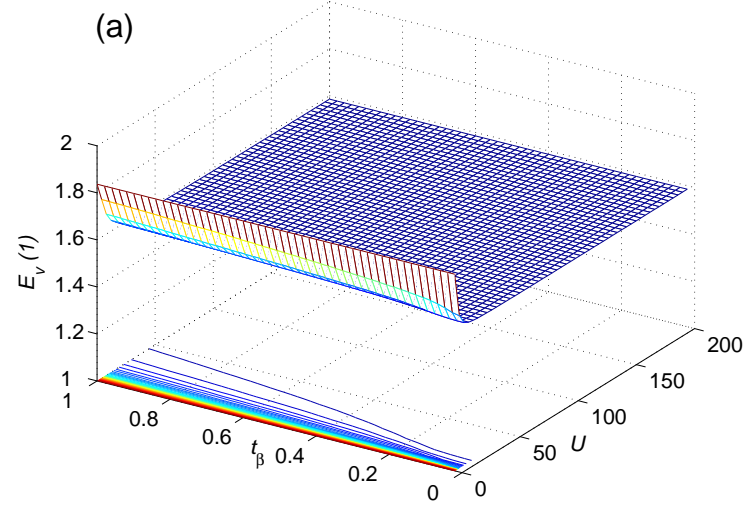

(c)

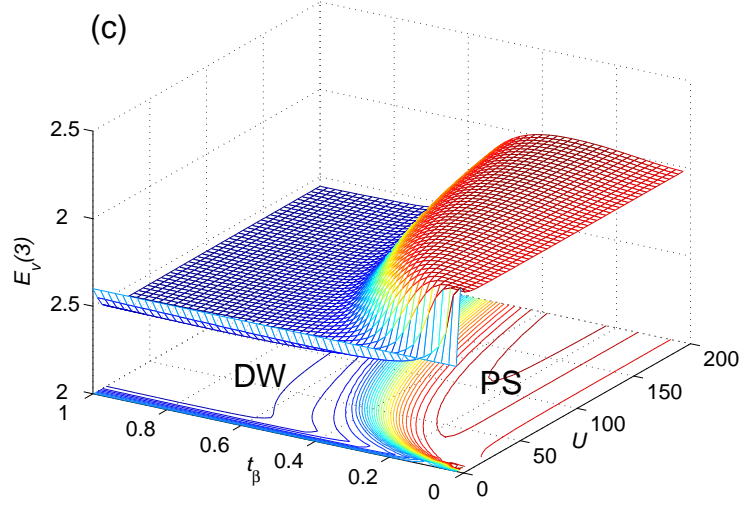

(b)

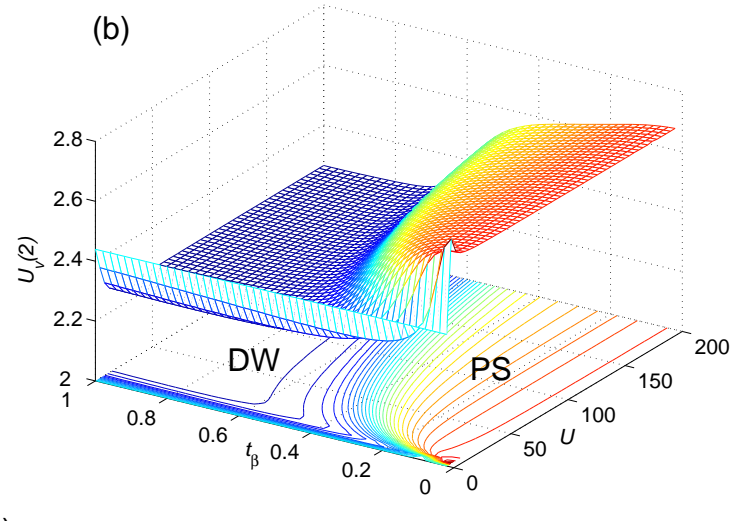

(b)

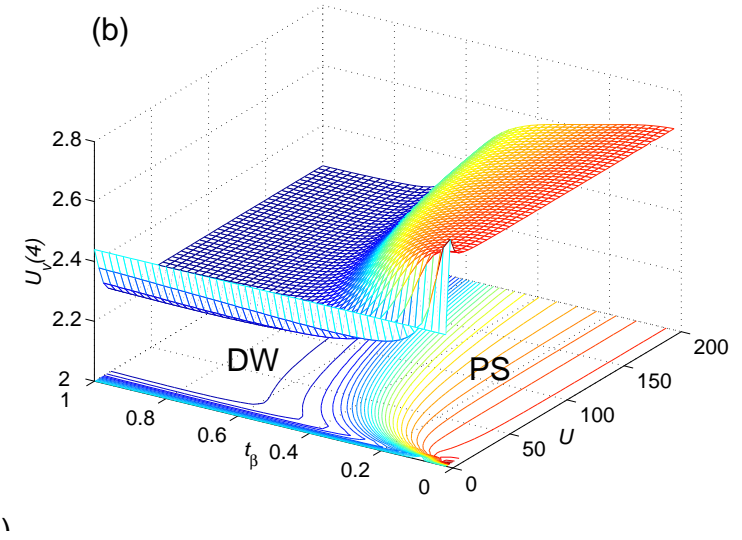

FIG. 1: (color online) The changes of symmetry in the ground state wave function is analyzed by considering the quantum correlation, i.e. entanglement, between local block and other parts of the system. Here $L=6, N_{\alpha}=N_{\beta}=2, l=2$, and the anti-periodic boundary conditions are assumed in order to avoid level-crossing in the ground state. Four figures correspond to different block size: $\mathrm{a}(l=1), \mathrm{b}(l=2), \mathrm{c}(l=3)$, and $\mathrm{d}(l=4)$.

\section{GROUND STATE ENTANGLEMENT}

In recent years, studies on the role of entanglement in the quantum critical behavior 23] have established a bridge between quantum information theory and statistical physics. [24, 25, 26] It is believed that the entanglement, as a kind of quantum correlation, can help us identify quantum phase transition in many-body systems. To have an intuitive picture of the global phase diagram, we must first compute the entanglement between a local block and the rest of the system. For the present model, the local states on each site have four possible configurations, denoted by

$$
\phi_{l}=|0\rangle,|\alpha\rangle,|\beta\rangle,|\alpha \beta\rangle ; l=1,2,3,4 .
$$

The Hilbert space associated with the $L$-site system is spanned by $4^{L}$ basis vectors. If we choose the periodic boundary conditions for $N=4 n+2$ and antiperiodic boundary conditions for $N=4 n$, where $n$ is an integer, the ground state is nondegenerate. Considering the reduced density matrix of a block of $l$ successive sites of the ground state

$$
\rho_{l}=\operatorname{tr}_{r}|\Psi\rangle\langle\Psi|,
$$

the von Neumann entropy $E_{v}(l)$, i.e.

$$
E_{v}(l)=-\operatorname{tr}\left[\rho_{l} \log _{2}\left(\rho_{l}\right)\right]
$$

measures the entanglement between the $l$ sites and the $L-l$ sites of the system. Like the well known fact in classical optics that the three-dimensional image of one object can be recovered from a small piece of holograph due to the interference pattern of the reflected light beams from it, quantum superposition principle also allows us to see a global picture of the system from its local part $[25,26]$. As was shown for some typical models in condensed matter physics, such as the extended Hubbard model [26], the entanglement of the ground state can give us a global view of the phase diagram.

¿From this point of view, we show a three-dimensional diagram and its contour map of the entanglement with block size $l=1,2,3,4$ for 6 -site system with $N_{\alpha}=N_{\beta}=$ 2 in Fig. 1. It has been pointed out that in the extended 


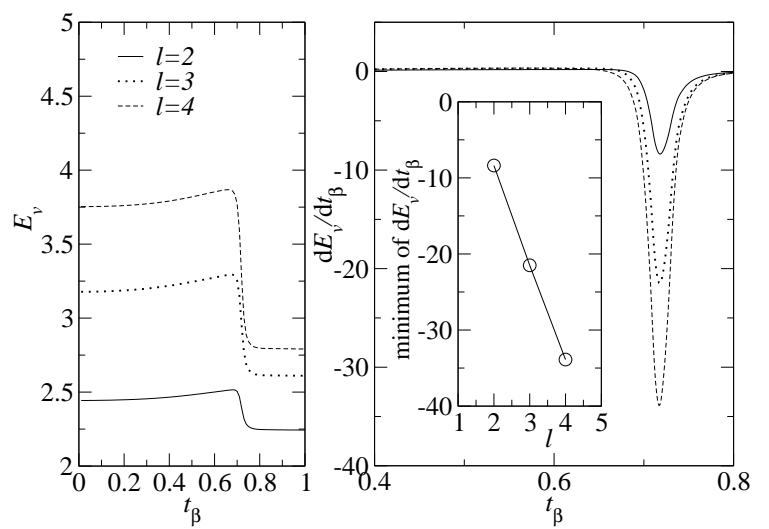

FIG. 2: The entanglement (left) and its first derivative (right) as a function of $t_{\beta}$ for various block size and a specified $U=$ 200. The inset shows the scaling behavior of the minimum point of $d E_{v}(l) / d t_{\beta}$ at the critical point. Here $L=10, N_{\alpha}=$ $N_{\beta}=4$.

Hubbard model, the single-site entanglement can distinguish the three main phases in the ground state. The reason is that the density distributions of the different modes in the reduced density matrix of a single site, such as the double occupancy, in the extended Hubbard model are sensitive in the quantum phase transitions. However, from Fig. 1(a), the single-site entanglement in AHM is rather trivial in the large $U$ region. It is not difficult to understand this phenomena. For the present model, the reduced density matrix of a single site has a simple form, [26, 29],

$$
\rho_{1}=z|0\rangle\left\langle 0\left|+u^{+}\right| \alpha\right\rangle\left\langle\alpha\left|+u^{-}\right| \beta\right\rangle\langle\beta|+w| \alpha \beta\rangle\langle\alpha \beta|,
$$

in which $z, u^{+}, u^{-}$, and $w$ are the density distributions for different local states, and can be calculated as

$$
\begin{aligned}
w & =\left\langle n_{\alpha} n_{\beta}\right\rangle=\operatorname{tr}\left(n_{\alpha} n_{\beta} \rho_{1}\right), \\
u^{+} & =\left\langle n_{\alpha}\right\rangle-w, u^{-}=\left\langle n_{\beta}\right\rangle-w, \\
z & =1-u^{+}-u^{-}-w .
\end{aligned}
$$

In the large $U$ limit, the double occupancy of two atoms on a single site is forbidden, i.e. $w \simeq 0$. Then for a finite system with periodic or anti-periodic boundary conditions, $\left\langle n_{\alpha}\right\rangle$ and $\left\langle n_{\beta}\right\rangle$ are constants. This fact leads to a constant single-site entanglement during the evolution of $t_{\beta}$ in the large $U$ region (Fig. 1(a)). For the case of $n_{\alpha}=1 / 3, n_{\beta}=1 / 3, E_{v}(1)$ has the value $\log _{2} 3$, so the single-site entanglement is insensitive to the phase transition from DW to PS. This property is very similar to the single-site entanglement in spin models [27] and the ionic Hubbard model. [28]

Clearly, the transition from DW to PS is intrinsically related to the change of density distribution of one species of atoms on the lattice. In order to contain enough information of the density-density correlation from the point of view of the entanglement, more sites should be included into the block. According to this point, we show the two-site entanglement as a function of $t_{\beta}$ and $U$ in Fig. 1 (b), from which we immediately notice two different regions: one is an altiplano marked with warm color (denoted by "PS" in the contour map of Fig. 1(b)), while the other is a plain with cold color (denoted by "DW" in contour map of Fig. 1 (b). Taking into account the known fact of the two limiting cases of this model, such an obvious difference witnesses the critical phenomenon between two universal classes.

In order to understand this obvious difference of the two-site entanglement in two phases, let us have a look at the structure of the corresponding reduced density matrix. For the AHM, the total numbers of $\alpha$ atoms and $\beta$ atoms are good quantum numbers, which leads to the fact that for arbitrary block size $l$, there is no coherent superposition of local states with different values of $N_{\alpha}$ and $N_{\beta}$ in the reduced density matrix. That is, the reduced density matrix must have the block-diagonal form classified by both $N_{\alpha}(l)$ and $N_{\beta}(l)$, i.e.

$$
\rho_{l}=\operatorname{diag}\left\{\rho_{l}(0,0), \rho_{l}(1,0), \rho_{l}(0,1), \ldots, \rho_{l}(l, l)\right\}
$$

where $\rho_{l}\left(n_{a}, n_{b}\right)$ is a matrix which has $n_{a} \alpha$ atoms and $n_{b} \beta$ atoms. According to the definition of von Neumann entropy (hence the entanglement), its magnitude is really determined by the distribution of the eigenvalues of the reduced density matrix. That is, the more uniformly distributed the eigenvalues, the higher the entropy. In the PS phase, elements in the reduced density matrix related to the basis $|\beta \beta\rangle$, which denotes two $\beta$ atoms congregate together, are finite, while in the DW region, they are almost zero. This fact leads to a larger entanglement with a block size larger than 2 in the PS phase, but otherwise in the DW phase. So the transition introduces a significant change into the value of the entanglement, and vice versa. From Fig. 1 (c), we can see that $E_{v}(3)$ shares similar properties with $E_{v}(2)$. On the other hand, since the ground state is translational invariant, the entanglement satisfies the equation $E_{v}(l)=E_{v}(L-l)$, Therefore, we have the same figures of $E_{v}(2)$ and $E_{v}(4)$ (Fig. 11(d)) for the 6 -site system.

Moreover, in the region $l \in[0, L / 2]$ the entanglement is a non-decreasing function of $l$, as is shown in Fig. 2 for a 10-site system with $N_{\alpha}=N_{\beta}=4$ and $U=200$. Therefore its first derivative develops a minimum at the critical point, as we can see from Fig. 2. Moreover, as the block size increases, the minimum point becomes sharper and sharper, exhibits a scaling behavior as shown in the inset of Fig. 2 i.e., $d E_{v}(l) / d t_{\beta} \propto-l$ around the critical $t_{\beta}$.

\section{CHARGE ORDER PARAMETER AND PHASE DIAGRAM}

Though the entanglement can give us useful information about the phase diagram, the dominating configurations in different phases remains unknown. So it is important to study the structure factor in competing phases. 


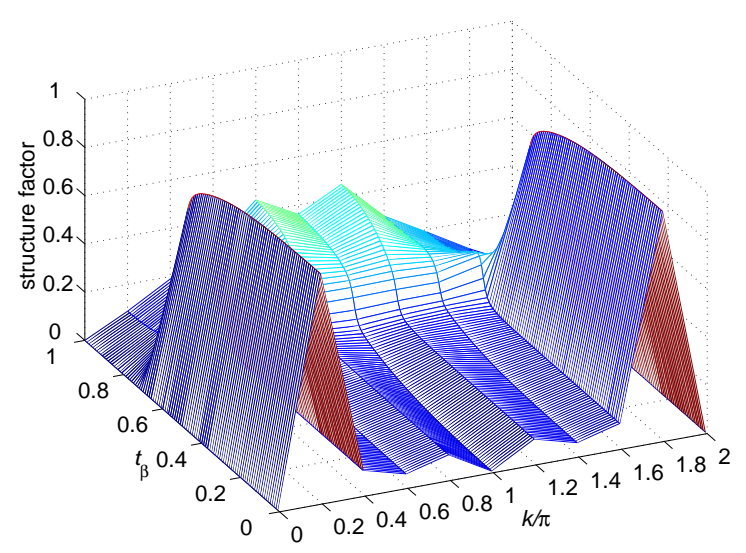

FIG. 3: (color online) The structure factor of DW as a function of $t_{\beta}$ and various modes, i.e. quantized momentum. Here $L=10, N_{\alpha}=N_{\beta}=4, U=200$.

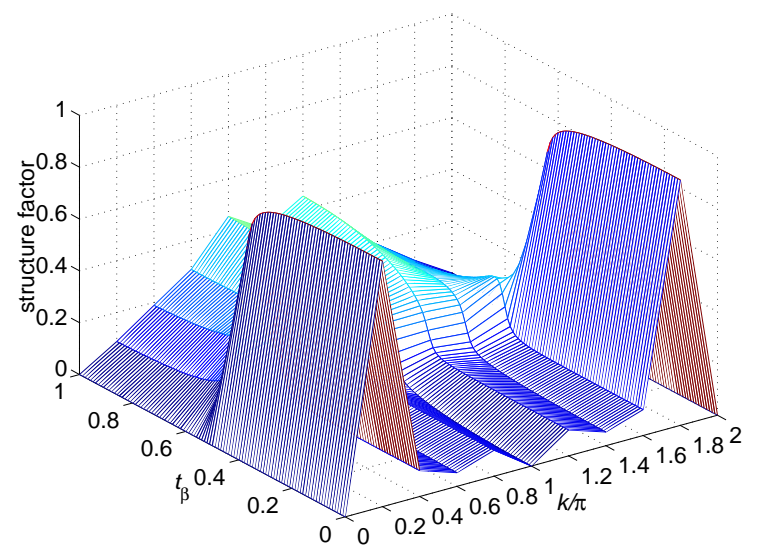

FIG. 4: (color online) The structure factor of DW as a function of $t_{\beta}$ and various modes, i.e. quantized momentum. Here $L=10, N_{\alpha}=N_{\beta}=4, U=50$.

Taking into account that the dominating configuration of $\beta$ atoms is quite different in two phases, we introduce the following structure factor of DW of $\beta$ atoms

$$
S_{\mathrm{CDW}}(q)=\frac{1}{L} \sum_{j l}\left[e^{i q(j-l)}\left(\left\langle n_{j, \beta} n_{l, \beta}\right\rangle-\left\langle n_{\beta}\right\rangle^{2}\right)\right],
$$

where $q=2 n \pi / L, n=0,1, \cdots, L$. In Fig. 3, we show that the structure factor as a function of $t_{\beta}$ for different modes for a system with $L=10$ and a relatively large $U=200$. The figure shows an obvious competition between the two modes. In the small $t_{\beta}$ limit, i.e., when $\beta$ atom has very heavy mass, $S_{\mathrm{CDW}}(q=2 \pi / L)$ dominates, which indicates phase separation in this region [20]. A careful scrutiny of the ground-state wavefunction finds that the configuration

$$
|\beta, \beta, \beta, \beta, \circ, \circ, \circ, \circ, \circ, \circ\rangle
$$

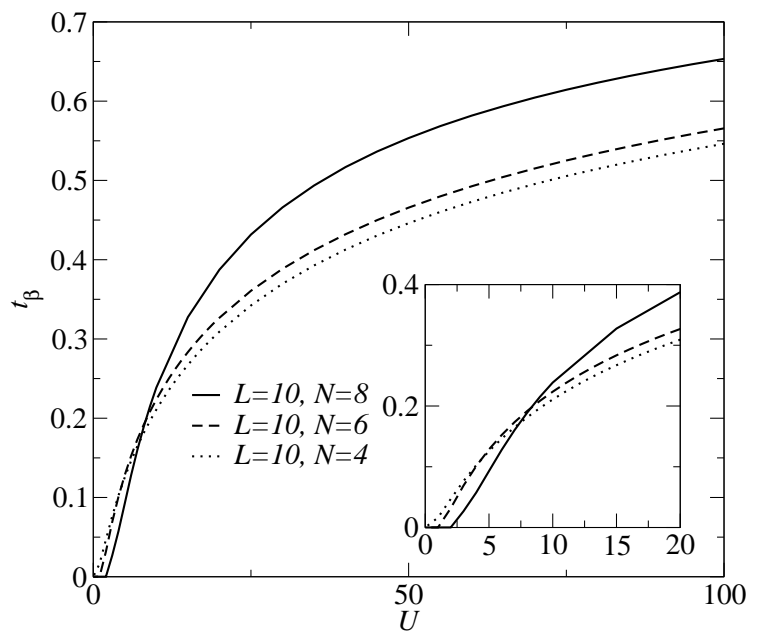

FIG. 5: (color online) The ground phase diagram in the $U-t_{\beta}$ plane in large $U$ case. The dominating configuration in the up-region is DW and below the boundary line it is PS. Here $L=10, N_{\alpha}=N_{\beta}$.

of $\beta$ atoms is dominant. It is not difficult to interpret this result. In the small $t_{\beta}$ limit, the major contribution to the ground-state energy comes from the $\alpha$ atoms. So in order to have a lower energy, they need more free space because the energy of particles inside the Fermi surface is $-2 \cos (k), k \propto 1 / L$. Then $\beta$ atoms will be pushed by $\alpha$ atoms to form clusters and phase separation occurs. For a finite size system, the translational symmetry is still preserved, while in the thermodynamic limit, especially in high dimensions, the symmetry in the ground state might be broken due to the very high potential energy between different configurations of $\beta$ atoms. Then the system will be separated into two distinct regions macroscopically. One is the solid-like region of $\beta$ atoms, while the other is the liquid-like region of $\alpha$ atoms. The latter can be described by a model of $N_{\alpha}$ atoms trapped in an infinite potential well with length $L-N_{\beta}+1$. The ground state is insulating and the energy is simply

$$
E_{0} \simeq-2 \sum_{j=1}^{N_{\alpha}} \cos \left(\frac{j \pi}{L-N_{\beta}+1}\right) .
$$

While if $t_{\beta} \rightarrow 1, S_{\mathrm{CDW}}(q=N \pi / L)$ exceeds $S_{\mathrm{CDW}}(q=$ $2 \pi / L)$, which implies that $\beta$ atoms distribute uniformly on the optical lattice. Then together with $\alpha$ atoms, the ground state becomes the so called DW state, which can be regarded as a solution of $\alpha$ and $\beta$ atoms, as shown by the configuration

$$
|\beta, \alpha, \circ, \beta, \alpha, \beta, \circ, \alpha, \beta, \alpha\rangle .
$$

of $\alpha$ and $\beta$ atoms. In its limiting case $t_{\beta}=1$, the model goes back to the traditional Hubbard model whose excitation spectrum is gapless, so the system is a conductor away from half-filling [17]. Therefore, different configurations dominate in different regions and the competition 
between them leads to a critical phenomenon. According to this criterion, we can use the intersection of the structure factor of two modes to determine the transition point on the $U-t_{\beta}$ plane for a finite system. We plot the phase diagram on the $U-t_{\beta}$ plane in Fig. 5 for a 10-site system with different filling $N_{\alpha}=N_{\beta}=4,6,8$.

However, the results for a finite system is rather qualitative. In order to have quantitative results for a real system, scaling analysis is crucial. For this purpose, we first estimate the scaling behavior of the ground-state energy in the critical region by the ED and DMRG method. 30. In Fig. 6, we show the scaling behavior of the groundstate energy at a given density $n=2 / 3$. Results are obtained for systems with open boundary conditions via the DMRG method in which up to 150 states are kept in the finite algorithm. It is evident that the limiting energy is approached linearly with $1 / L$. A relation of the form

$$
E_{0}(N)=E(\infty)+a / L
$$

where $a$ is a constant, holds quite accurately in a large $U$ region. Since the quantum critical phenomena is related to the singularity in ground-state energy, the $1 / L$ correlation in Eq. (17) actually implies that the phase boundary bears a similar scaling behavior. Based on this consideration, we take $n=2 / 3$ as an example to show the scaling behavior of the phase boundary for both open and anti-periodic boundary conditions in Fig. 7.

It has been shown that, based on the variation principles, one can obtain the lower and upper bounds of the phase boundary with different boundary conditions, such as periodic, anti-periodic, and open boundaries. From Fig. 7, it is clearly shown that data with APBC give a lower bound while data with OBC give an upper bound on the transition point. Moreover, the extrapolated data based on the $1 / L$ scaling of the two approaches for an infinite system agree with each other. This phenomenon is consistent with the fact that the physics in a real system should be independent of the boundary conditions. Moreover, we can also estimate errors in our extrapolation. We presented a final phase diagram with error bars smaller than the size of the symbols in Fig. 8.

In the small $U$ region, we can see that there is a critical $U$ on the $U$-axis for the density $n=2 / 3$. However, if $n$ is reduced, the critical $U$ tends to zero. In the low density limit, the phase boundary scales like $t_{\beta} \propto U^{2}$ in the small $U$ region, which agrees with the results obtained by the Bosonization method [32] excellently.

In the large $U$ region, the critical $t_{\beta}$ increases as $U$ increases. We take a system of $L=12, N_{\alpha}=N_{\beta}=4$ as an example, and show the $1 / U$ behavior of the phase boundary in Fig. 9. From the figure, we can see that the critical $t_{\beta}$ is proportional to $1 / U$ in the large $U$ limit. Moreover, Fig. 9 manifests that $U$ will be saturated in the infinite $U$ limit. That is, for a given concentration, there exists a saturation $t_{\beta}^{s}$ above which the phase separation will never happen regardless how large the on-site $U$ is. Based on these physical intuition, the boundary

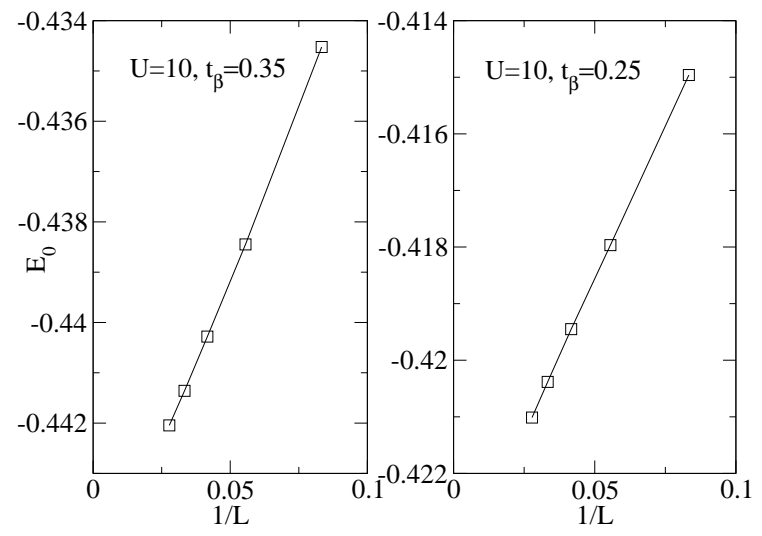

FIG. 6: The scaling behavior of the ground-state energy for two points near to the both sides of the critical point. Here $n=2 / 3$.

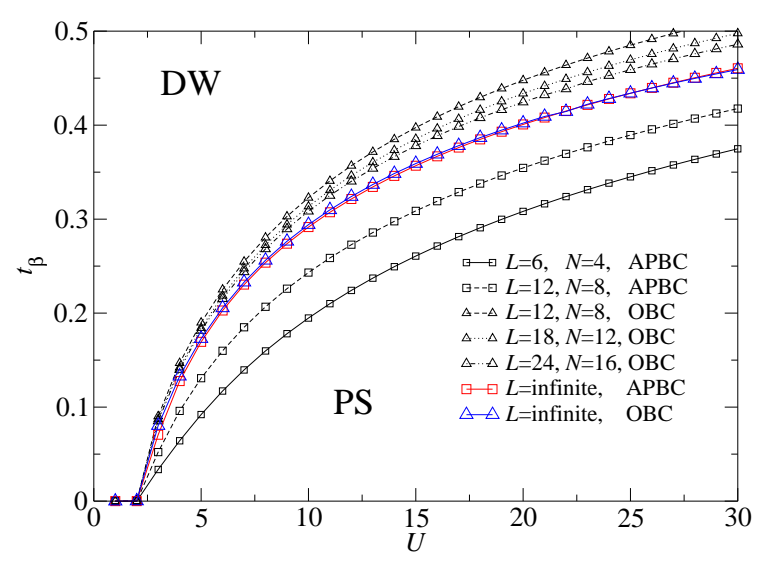

FIG. 7: The scaling behavior of the phase boundary for both open boundary conditions (triangular lines) and anti-periodic boundary conditions (square lines). Here $n=2 / 3$.

line satisfies the relation

$$
t_{\beta}=t_{\beta}^{s}+C / U,
$$

where $C$ is a constant, and both $t_{\beta}^{s}$ and $C$ depend on the filling conditions.

\section{SINGLE-HOLE PROBLEM}

In this section, we give a rigorous proof that even for the case of one hole doping away from half-filling, the DW state is unstable to the PS state in the infinite $U$ limit. If $U$ is very large, the critical point then is approached linearly with $1 / U$.

We first consider an odd-site sample with $L=2 N_{\alpha}+$ $1, N_{\alpha}=N_{\beta}$ and infinite $U$. The space of DW is spanned by $2 L$ basis:

$$
\left|e_{i}\right\rangle=\left|\alpha_{1}, \beta_{2}, \cdots \circ_{i} \ldots, \alpha_{L-1}, \beta_{L}\right\rangle, i \in[1, L]
$$




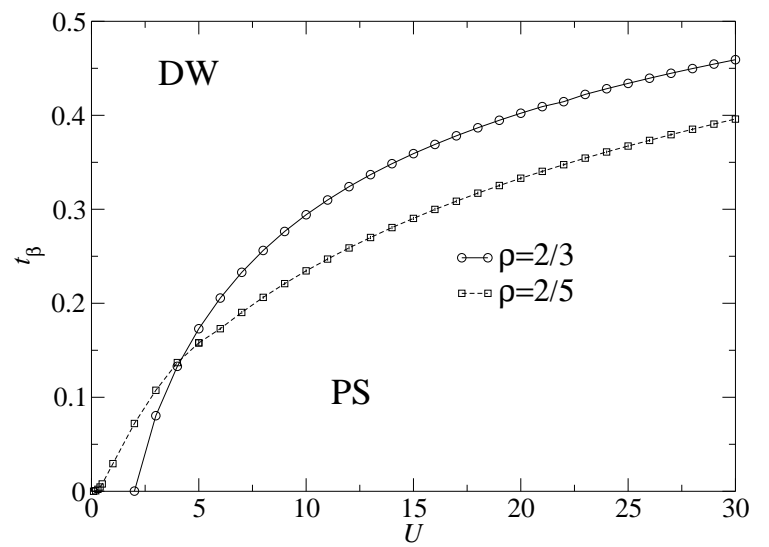

FIG. 8: The ground-state phase diagram of the AHM for two filling condtions: $n=2 / 3,2 / 5$.

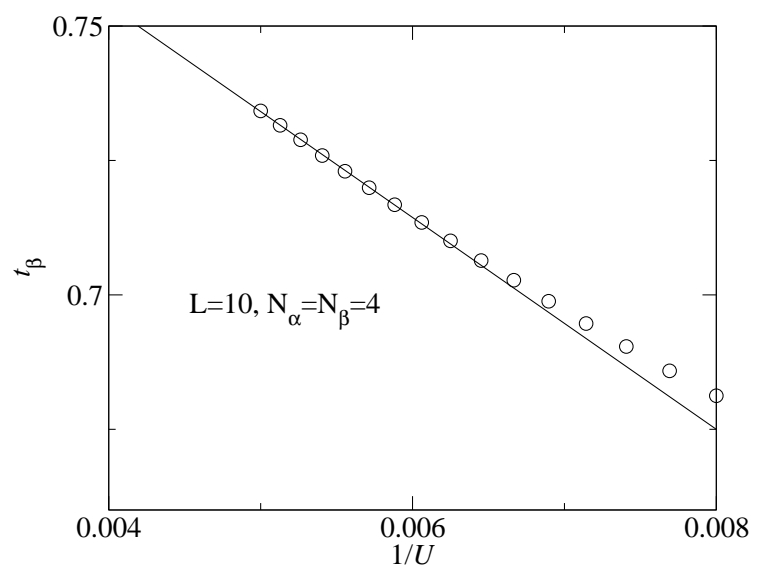

FIG. 9: $1 / U$ behavior of the phase boundary in the large $U$ region, as exemplified by a system with $L=12, N_{\alpha}=N_{\beta}=4$.

and

$$
\left|e_{i}\right\rangle=\left|\beta_{1}, \alpha_{2}, \cdots \circ_{i-L} \ldots, \beta_{L-1}, \alpha_{L}\right\rangle, i \in[L+1,2 L],
$$

where $\circ_{i}$ denotes a hole at site $i$. Then the Hamiltonian

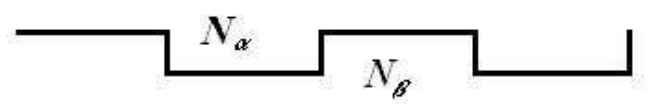

FIG. 10: The periodical square potential wells used to describe the dynamics of a hole in demixed phase.
(1) becomes

$$
\left(\begin{array}{ccccccc}
0 & -1 & 0 & 0 & \ldots & 0 & -t_{\beta} \\
-1 & 0 & -t_{\beta} & 0 & \ldots & 0 & 0 \\
0 & -t_{\beta} & 0 & -1 & \ldots & 0 & 0 \\
0 & 0 & -1 & 0 & \ldots & 0 & 0 \\
\vdots & \vdots & \vdots & \vdots & \ddots & \vdots & \vdots \\
0 & 0 & 0 & 0 & \ldots & 0 & -1 \\
-t_{\beta} & 0 & 0 & 0 & \ldots & -1 & 0
\end{array}\right) .
$$

It can be solved exactly. The whole energy spectra of the system are given by

$$
\begin{aligned}
E_{ \pm} & = \pm \sqrt{1+t_{\beta}^{2}+2 t_{\beta} \cos \left(k_{j}\right)}, \\
k_{j} & =j \pi / L, j=0,1, \ldots, L-1
\end{aligned}
$$

The ground-state wavefunction for arbitrary odd $L$ is

$$
\left|\Psi_{0}\right\rangle=\frac{1}{\sqrt{2 L}} \sum_{i=1}^{2 L}\left|e_{i}\right\rangle,
$$

with the eigenenergy $E_{\mathrm{DW}}=-1-t_{\beta}$.

However, in the case of completely demixed phase, the whole space is spanned by $L(L-1)$ basis. The Hamiltonian then describes the problem of single particle motion in periodic square potential wells (see Fig. 10) with different hoping integrals in different region. Precisely, the Hamiltonian becomes

$$
H=-\sum_{j, \delta} d_{j}^{\dagger} d_{j+\delta},
$$

where $d_{j}^{\dagger}$ and $d_{j}$ are hole creation and annihilation operators in each potential well, and

$$
H=-t_{\beta} \sum_{j, \delta} d_{j}^{\dagger} d_{j+\delta}
$$

elsewhere. The ground state of this Hamiltonian in the thermodynamic limit is identical to the ground state of

$$
H=\left\{\begin{array}{cc}
-2+p^{2}, & 0<x<N_{\alpha} ; \\
-2 t_{\beta}+t_{\beta} p^{2}, & N_{\alpha}<x<N_{\beta},
\end{array}\right.
$$

with periodic boundary conditions

$$
\Psi(x)=\Psi\left(x+N_{\alpha}+N_{\beta}\right) .
$$

For the latter, a bound state always exists for arbitrary well depth $2-2 t_{\beta}$. Then if $N_{\alpha}=N_{\beta} \rightarrow \infty$, the groundstate energy is simply -2 , which is obviously smaller than $E_{\mathrm{DW}}=-1-t_{\beta}$. For a finite system except $L=3$, it can also be shown that $E_{\mathrm{PS}}(L)<E_{\mathrm{DW}}(L)$. For example if $L=5, N_{\alpha}=N_{\beta}=2$, we have

$$
E_{\mathrm{PS}}(L=5)=-\sqrt{2+2 t_{\beta}^{2}} .
$$

Therefore, in the infinite $U$ limit, the DW state is unstable to the PS state. Such a rigorous result is also valid 
for a system of two species of hard-core bosonic atoms and Bose-Fermi mixtures with different hoping integrals in optical lattices.

When the on-site $U$ is very large but not infinite, the Hamiltonian (11) can be approximated by [13]

$$
\begin{aligned}
H= & -\sum_{j=1}^{L} \sum_{\delta= \pm 1} \sum_{\sigma} t_{\sigma} c_{j, \sigma}^{\dagger} c_{j+\delta, \sigma} \\
& +J \sum_{j=1}^{L}\left[\mathbf{S}_{j} \cdot \mathbf{S}_{j+1}+\delta S_{j}^{z} S_{j+1}^{z}-\mu n_{j} n_{j+1}\right]
\end{aligned}
$$

in which

$$
J=\frac{4 t_{\alpha} t_{\beta}}{U}, \quad \delta=\frac{\left(t_{\alpha}-t_{\beta}\right)^{2}}{2 t_{\alpha} t_{\beta}}, \quad \mu=\frac{t_{\alpha}^{2}+t_{\beta}^{2}}{8 t_{\alpha} t_{\beta}} .
$$

Clearly if $t_{\alpha}=t_{\beta}$, the above Hamiltonian becomes the $t-J$ model. The ground state of the $t-J$ model with a single-hole doping becomes the Nagaoka ferromagnetism [31] if the contribution from the kinetic energy in Eq. (27) exceeds that from the spin-spin antiferromagnetic interaction. In order to study the condition of PS, we first suppose that the ground state of the system is phase separated. Then the ground-state energy can be approximated by

$$
E_{\mathrm{PS}} \simeq-2+\frac{(L-6)(1+\delta) J}{4},
$$

On the other hand, if the ground state is in the DW state, the ground-state energy can be approximated by that of the XXZ chain. For the latter, the ground-state energy per bond has the form 33.

$$
e_{\mathrm{XXZ}}=\frac{1+\delta}{4}-\sinh \phi\left[\frac{1}{2}+2 \sum_{n=1}^{\infty} \frac{1}{e^{2 n \phi}+1}\right]
$$

where $\cosh \phi=1+\delta$. Then the ground-stat energy of the DW phase becomes

$$
E_{\mathrm{DW}} \simeq-1-t_{\beta}+(L-2) J e_{\mathrm{Xxz}} .
$$

Here, in both $E_{\mathrm{DW}}$ and $E_{\mathrm{PS}}$, the finite-size correction to the ground-state energy is not taken into account, so the critical value is estimated approximately. Despite of this, the qualitative behavior of critical point is clear, i.e.

$$
t_{\beta} \simeq 1-J L \sinh \phi\left[\frac{1}{2}+2 \sum_{n=1}^{\infty} \frac{1}{e^{2 n \phi}+1}\right]+O(1 / U),
$$

which means only if $U \gg L$, the DW state is unstable to PS state. For a given $L$, the phase boundary scales like

$$
t_{\beta}=1-C / U,
$$

in the large $U$ limit. Clearly, Eq. (33) is consistent with our previous result Eq. (18).

\section{DISCUSSIONS}

Obviously, unlike the PS in the $t-J$ model [15] and the extended Hubbard model [26], which is the consequence of attractive interaction between particles, the PS in the ground state of the AHM is driven by kinetic energy. Therefore, though our results are based on a onedimensional model, the underlying physics is quite general for systems in any dimension. That is, in the large $U$ limit, the dynamics of a system of two species of atoms at zero temperature is dominated by the light atoms. In order to have a lower energy, they need more free space to move. This mechanism forces heavy atoms to congregate together, so the latter becomes a solid-like object. In experiment, two separated regions are expected to be witnessed macroscopically. However, when $t_{\beta} \rightarrow t_{\alpha}$, the dynamics of heavy atoms is comparable to that of light atoms, the exchange interaction drives the system into a DW state. Therefore, if we consider the PS state as a classical phase containing solid-like order and the DW state as a quantum region with liquid properties in the whole system, the transition reported in our work is just an example of a crossover from the classical region to the quantum region.

Such an interesting transition is expected to be observed in the on-going experiments on optical lattices. We take a system consisting of two species of atoms (such as ${ }^{6} \operatorname{Li}(\alpha)$ and ${ }^{40} \mathrm{~K}(\beta)$ with $\left.m_{\beta} / m_{\alpha} \simeq 20 / 3\right)$ as an example. Since the typical scattering length for alkaline atoms ranges from 40 to $100 a_{\text {Bohr }}$ 34, 35], and laser wavelength $\lambda=852 \mathrm{~nm}[3]$. Then from Eq. (8) we roughly estimate that PS phase can be observed when $v>0.4$ according the phase diagram in Fig. 8

\section{SUMMARY}

In summary, we have investigated the ground-state phase diagram of two species of fermionic atoms trapped in one-dimensional optical lattice. By using the ED method, we computed the block-block entanglement between a local block and rest part for a small system. We obtained an intuitive picture of phase diagram of the ground state and found that the entanglement in the PS region is in general larger than that in the DW region for a finite system. Its first derivative develops a sharp downward peak and shows scaling behavior at the critical point. We also analyzed the structure factor of the DW of $\beta$ atoms by the ED and DMRG method, and found that the competition between two different configurations in the ground-state wavefunction leads to a phase transition at the critical point. The global phase diagram was obtained from the careful scaling analysis for various-size systems and different boundary conditions. Therefore, we results firstly gave a quantitative description of the ground-state phase transition of the AHM away from the half-filling. Furthermore, we gave a rigorous proof that even for the case of a single hole doping, the DW state is 
unstable to the PS in the infinite $U$ limit. Such a rigorous conclusion clarifies the physical picture of the phase separation.

\section{ACKNOWLEDGEMENTS}

We thank Y. G. Chen, Z. G. Wang, X. G. Wen, G. M. Zhang for helpful discussions, and Miss Daisy Lin for critical reading of the manuscript. H. Q. Lin thanks Research Center of Quantum Control at Fadan University for its hospitality where part of this work was carried out. This work is supported in part by the Earmarked Grant for Research from the Research Grants Council of HKSAR, China (Project CUHK 401504).
[1] I. Bloch, Nature Physics, 1, 23 (2005).

[2] M. Holland, et al, Phys. Rev. Lett. 87, 120406 (2001); Y. Ohashi and A. Griffin, Phys. Rev. Lett. 89, 130402 (2002); C. A. Regal, et al, Phys. Rev. Lett 92, 040403 (2004); M. W. Zwierlein, et al, Phys. Rev. Lett 92, 120403 (2004); M. W. Zwierlein, et al, Nature 435, 1047 (2005).

[3] M. Greiner et al, Nature 415, 39 (2002);

[4] O. Mandel, et al, Nature 425, 937 (2003); O. Mandel, et al, Phys. Rev. Lett. 91, 010407 (2003).

[5] C. Chin et al, Science 305, 1128 (2004); J. Kinast, et al, Science 307, 1296 (2005); M. Köhl, et al, Phys. Rev. Lett. 94, 080403 (2005); M. Bartenstein et al, Phys. Rev. Lett. 94, 103201 (2005).

6] D. Jaksch, et al, Phys. Rev. Lett. 81, 3108 (1998).

[7] W. Vincent Liu, F. Wilczek, and P. Zoller, Phys. Rev. B 70, 033603 (2004).

[8] W. Hofstetter, J. I. Cirac, P. Zoller, E. Demler, and M. D. Lukin, Phys. Rev. Lett. 89, 220407 (2002).

[9] M. A. Cazalilla, A. F. Ho, and T. Giamarchi, Phys. Rev. Lett. 95, 226402 (2005).

[10] L. M. Duan, Phys. Rev. Lett. 95, 243202 (2005); L. M. Duan, Europhys. Lett. 67, 721 (2004).

[11] R. B. Diener and T. L Ho, Phys. Rev. Lett. 96, 010402 (2006).

[12] U. Brandt, J. Low Temp. Phys. 84, 477 (1991).

[13] G. Fáth, Z. Domański, and R. Lemański, Phys. Rev. B 52, 13910 (1995).

[14] C. A. Macedo and A. M. C. de Souza, Phys. Rev. B 65, 153109 (2002).

[15] V. J. Emery, S. A. Kivelson, and H. Q. Lin, Phys. Rev. Lett. 64, 475 (1990).

[16] F. Paesani, F. A. Gianturco and K. B. Whaley, Europhys. Lett. 56, 658 (2001), and the references therein.

[17] J. Hubbard, Proc. R. Soc. London A 276, 238 (1963).

[18] E. H. Lieb and F. Y. Wu, Phys. Rev. Lett. 20, 1445
(1968).

[19] L. M. Falicov and J. C. Kimball, Phys. Rev. Lett. 19, 997 (1969).

[20] P. Lemberger, J. Phys. A 25, 715 (1992).

[21] J. K. Freericks and V. Zlatic, Rev. Mod. Phys. 75, 1333 (2003).

[22] T. Kennedy and E. Lieb, Physica A 138:320 (1986); J. Lebowitz and N. Macris, Rev. Math. Phys. 6:927 (1994).

[23] S. Sachdev, Quantum Phase Transitions, (Cambridge University Press, Cambridge, UK, 2000).

[24] For examples, A. Osterloh, et al, Nature 416, 608 (2002); T. J. Osborne and M.A. Nielsen, Phys. Rev. A 66, 032110(2002); S. J. Gu, et al, Phys. Rev. A 68, 042330 (2003).

[25] G. Vidal, et al, Phys. Rev. Lett. 90, 227902 (2003); J. I. Latorre, et al, Quant. Inf. and Comp. 484 (2004).

[26] S. J. Gu, S. S. Deng, Y. Q. Li, and H. Q. Lin, Phys. Rev. Lett. 93, 086402 (2004); S. S. Deng, S. J. Gu, and H. Q. Lin, Phys. Rev. B 74, 045103 (2006).

[27] S. J. Gu, G. S. Tian, and H. Q. Lin, New. J. Phys. 8, 61 (2006).

[28] Ö. Legeza and J. Sólyom, Phys. Rev. Lett. 96, 116401 (2006)

[29] P. Zanardi, Phys. Rev. A 65, 042101 (2002).

[30] S. R. White, Phys. Rev. Lett. 69, 2866 (1992); U. Schollwöck, Rev. Mod. Phys. 77, 259 (2005).

[31] Y. Nagaoka, Phys. Rev. 147, 392 (1966).

[32] Z. G. Wang, S. J. Gu, and Y. G. Chen, Phys. Rev. 75, 165111 (2007).

[33] C. N. Yang and C. P. Yang, Phys. Rev. 150, 321 (1966); 150, 327 (1966).

[34] M. Olshanii, Phys. Rev. Lett. 81, 938 (1998).

[35] M. Bartenstein, et al, Phys. Rev. Lett. 94, 103201 (2005). 